\title{
Hipertensão Portopulmonar e Gravidez
}

\author{
Portopulmonary Hypertension and Pregnancy
}

Pérsio Godoy, Guilherme Milhomem Martins, Márcio Weissheimer Lauria

\section{RESUM0}

A gravidade da coexistência de hipertensão pulmonar e gravidez está bem estabelecida. A hipertensão arterial pulmonar constitui condição com elevado risco de morte materna no final da gravidez e pós-parto. Pacientes portadores de hipertensão portal de várias etiologias podem desenvolver hipertensão arterial pulmonar (hipertensão portopulmonar), sendo a maioria dos casos relatados na cirrose hepática, entretanto uns poucos casos foram descritos na hipertensão portal não cirrótica. São apresentados o quadro clínico e anatomopatológico em dois casos de hipertensão portopulmonar e gravidez. Tratava-se de pacientes com 30 e 24 anos de idade, que desenvolveram insuficiencia cardiaca direita grave e choque no puerpério imediato, evoluindo rapidamente para o óbito. A necropsia demonstrou em ambos os casos fibrose nos espaços portais, correspondendo ao relatado na hipertensão portal idiopática, além de hipertensão pulmonar classificada como plexogênica.

PALAVRAS CHAVES: Hipertensão portal. Hipertensão pulmonar. Hipertensão portopulmonar. Complicações da gravidez. Mortalidade materna.

\section{Introdução}

A hipertensão pulmonar constitui relevante fator de risco na gravidez e no puerpério imediato, determinando alto índice de mortalidade materna (cerca de 25 a $50 \%$ ), na dependência da sua etiologia $^{1,2}$. Embora a hipertensão pulmonar represente contraindicação absoluta à gravidez, freqüentemente as pacientes são assintomáticas e o diagnóstico não é realizado no período pré-gestacional ou mesmo pré-natal, contrastando com a súbita e grave descompensação cardíaca no periparto, especialmente no puerpério imediato ${ }^{3}$.

Entre as causas de hipertensão arterial pulmonar suscetiveis de manifestação na gravidez, incluem-se a sindrome de Eisenmenger ${ }^{4}$, a hipertensão pulmonar primária ${ }^{5}$, o lupus eritematoso sistêmico $^{6}$ e a drepanocitose ${ }^{7}$, entre outras. Por seu turno, pouca referência é feita à presença de hipertensão portopulmonar na gravidez e não há

Faculdade de Medicina e Hospital das Clinicas da Universidade Federal de Minas Gerais

Correspondência:

Pérsio Godoy

Faculdade de Medicina da UFMG

Departamento de Anatomia Patológica e Medicina LegaL

Av. Prof. Alfredo Balena 190, 5o-andar

30130-100 - Belo Horizonte - MG

Fax: (31) 3222-3987 menção à gravidade da associação ${ }^{8}$.

A verificação de hipertensão pulmonar relacionada à hipertensão portal tem merecido maior atenção com o advento dos transplantes hepáticos, associada ou não à chamada síndrome portopulmonar, pois constitui contraindicação para o procedimento ${ }^{9-11}$.

Do ponto de vista morfológico as alterações vasculares pulmonares encontradas na hipertensão portopulmonar assemelham-se às descritas na hipertensão pulmonar primária, ambas classificadas como plexogênicas ${ }^{12,13}$.

\section{Relato dos Casos}

Caso 1: Paciente de 30 anos apresentou, no dia seguinte após o parto a fórcipe, súbito agravamento de dispnéia, dor torácica e tosse, iniciados quatro dias antes, além de edema periférico progressivo. A gestação teve curso normal, sem intercorrências clínicas, havendo relato de esplenectomia há dez anos, cuja indicação era desconhecida.

Apresentava palidez cutâneo-mucosa, anasarca, obesidade, cianose, dispnéia moderada a intensa; pressão arterial: 125/70 mmHg; freqüência cardiaca: $120 \mathrm{bpm}$, com presença de ter- 
ceira bulha; crepitações pulmonares bilaterais. O útero era palpável. Os exames complementares mostravam anemia macrocítica leve, leucocitose: $26.000 \mathrm{~mm}^{3}$, com desvio para esquerda, provas funcionais hepáticas normais, uréia: $82 \mathrm{mg} / \mathrm{dL}$, creatinina: $1,7 \mathrm{mg} / \mathrm{dL}$. A radiografia do tórax mostrava aumento da área cardíaca, com cefalização do fluxo, sem outros sinais de congestão pulmonar. O ECG demonstrou ritmo sinusal, sobrecarga do ventrículo direito e bloqueio de ramo direito do II grau.

Com as hipóteses diagnósticas de miocardiopatia periparto e embolia pulmonar, foi iniciado tratamento com digitálico, furosemida, captopril, heparina, aminofilina e oxigênio, sem resposta satisfatória. Apresentou piora do padrão respiratório, cianose, hiperfonese do componente pulmonar da segunda bulha cardíaca, sopro sistólico grau I/IV audível sobretudo na borda esternal esquerda e choque, com melhora após início de dopamina. No terceiro dia de internação ocorreu choque refratário, oligúria, pressão venosa central elevada ( $22 \mathrm{~cm}$ de $\mathrm{H}_{2} \mathrm{O}$ ), presença de crepitações teleinspiratórias nas bases, hipoxemia, acidose respiratória e leucocitose (40.000) com desvio para esquerda, evoluindo para óbito.

Na necropsia foram demonstrados: hipertensão pulmonar crônica (hipertrofia da camada média e discreta neoformação conjuntiva intimal em artérias de pequeno e médio calibre, além de múltiplas lesões plexiformes) (Figura 1); cor pulmonale crônico (hipertrofia discreta/moderada do miocárdio, em correspondência com a via de saída do ventrículo direito; dilatação do ventrículo e átrio direitos; átrio e ventrículo esquerdos sem alterações); esclerose hepatoportal (hipertensão portal idiopática): fibrose portal sistematizada, esclerose, compressão e redução de ramos da veia porta. Os ramos preservados mostravam hipertrofia da média; havia numerosos vasos venosos delgados e dilatados em plena fibrose. Encontravam-se também septos fibrosos portais delgados e incompletos, especialmente em áreas subcapsulares, sem constituírem "nódulos", hipotrofia do parênquima e distribuição irregular das veias centrais. Foram descritos também esplenectomia antiga, útero puerperal (650 g) e mamas da lactação.

Caso 2: Paciente de 24 anos previamente hígida, referia gestação sem intercorrências com parto normal e alta hospitalar no dia seguinte.

No terceiro dia pós-parto, iniciou com dispnéia aos mínimos esforços, edema generalizado e crise convulsiva. Apresentava cianose, hipotensão (pressão arterial: 90/70 $\mathrm{mmHg}$ ), freqüência cardíaca: $110 \mathrm{bpm}$, bulhas cardiacas rítmicas com hiperfonese e desdobramento da se- gunda bulha e sopro sistólico grau II/VI, em toda a borda esternal esquerda. Pulmões limpos com freqüência respiratória de $25 \mathrm{rpm}$. Hepatomegalia dolorosa ( $3 \mathrm{~cm}$ do RCD e a $7 \mathrm{~cm}$ do AX). Útero a 6 $\mathrm{cm}$ da cicatriz umbilical. Os exames complementares revelaram anemia e leucocitose discretas, plaquetas: $90.000 \mathrm{~mm}^{3}$, alterações leves da função hepática, uréia: $110 \mathrm{mg} / \mathrm{dL}$, creatinina: $1,2 \mathrm{mg} / \mathrm{dL}$. Radiografia de tórax: aumento da área cardiaca, especialmente do ventrículo direito, abaulamento do arco médio. ECG: ritmo sinusal, desvio do eixo para a direita, sobrecarga do VD e onda T negativa em AVF, DII, DIII e V1 a V6.

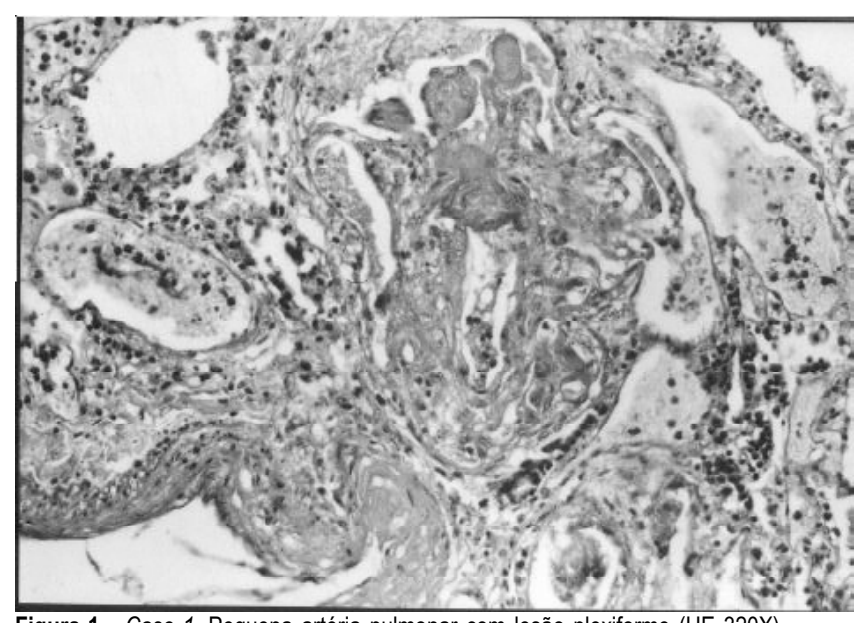

Figura 1 - Caso 1. Pequena artéria pulmonar com lesão plexiforme (HE 320X).

Levantadas as hipóteses diagnósticas de ICC, miocardiopatia periparto e tromboembolia pulmonar, recebeu tratamento com oxigênio, digitálico, diurético e heparina. Houve agravamento do quadro, com hipotensão transitória, ingurgitamento jugular, pressão venosa central muito elevada, cianose e oligúria. Apresentou crise convulsiva no nono dia após o parto. No décimo quinto dia pós-parto evoluiu com choque, queda progressiva do nível de consciência, hipoxemia e insuficiência respiratória. Ocorreu parada cárdio-respiratória, com retorno temporário por 15 minutos, em seguida surgiu fibrilação ventricular, sem resposta às manobras de reanimação. Óbito no $16^{\circ}$ dia pós-parto.

Na necropsia foram evidenciados: hipertensão pulmonar crônica (hipertrofia da média e neoformação conjuntiva intimal moderada/intensa em artérias de pequeno e médio calibre; lesões plexiformes e trombose de alguns pequenos vasos) (Figura 2); cor pulmonale crônico (hipertrofia do ventrículo direito, mais acentuada em sua via de saída; dilatação do átrio e ventrículo direitos; ventrículo e átrio esquerdo sem alterações). O peso do coração era de $380 \mathrm{~g}$, presença de hidropericár- 
dio (200 mL); fibrose hepática portal e periportal acentuada (Figura 3) (septos fibrosos delgados, em geral incompletos; hipertrofia da média e neoformação conjuntiva intimal em ramos da veia porta; espessamento da cápsula de Glisson, que envia septos ao parênquima subjacente, formando pequenos nódulos; proliferação pseudoductular e vascular em alguns espaços portobiliares); varizes discretas no terço inferior do esôfago, baço esclerocongestivo (peso: 350 g) e hidroperitônio (500 mL de líquido amarelo-citrino). Havia também congestão passiva e necrose hepática centro/mediolobular (Figura 3), rins do choque, gastrite hemorrágica aguda, congestão visceral generalizada, útero puerperal e mamas em lactação.

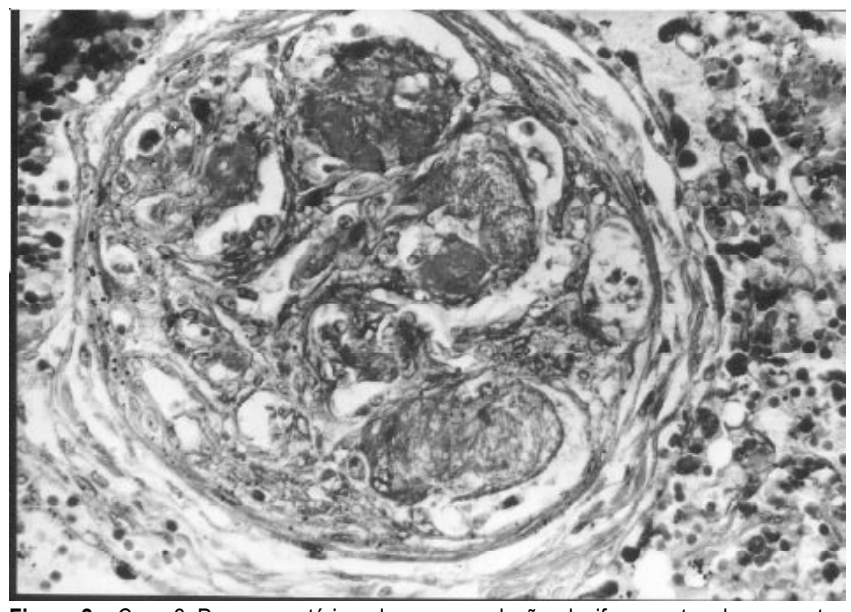

Figura 2 - Caso 2. Pequena artéria pulmonar com lesão plexiforme e trombos recentes (HE 640X).

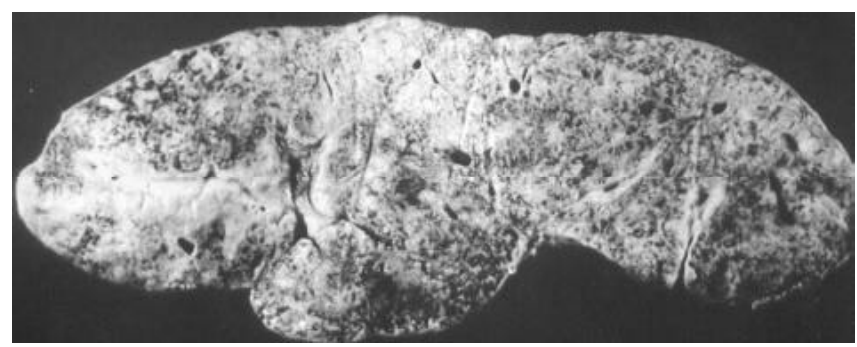

Figura 3 - Caso 2. Superfície de corte do fígado com fibrose portal (esclerose hepatoportal), nódulos subcapsulares incompletos; congestão e hemorragia (fígado cardíaco).

\section{Discussão}

Os casos relatados seguem a historia natural descrita quando há associação entre a hipertensão pulmonar e gravidez. As pacientes não apresentaram evidências de hipertensão pulmonar antes da gestação, porém desenvolveram insuficiência cardíaca grave no puerpério imediato, refratária ao tratamento instituído. A gravidez constituiu fator de exacerbação de hipertensão pulmonar prévia, tendo sido o diagnóstico feito após o óbito 5 .

Efetivamente, são bem conhecidas as graves repercussões da hipertensão pulmonar na vigência de gravidez, especialmente no período periparto, determinando com freqüência o óbito materno. Este fato justifica para alguns a contraindicação absoluta da gravidez, a interrupção precoce da gestação ou seu diagnóstico em tempo de serem instituídos cuidados, inclusive quanto à anestesia, na época do parto ${ }^{14}$.

Considerando a sobrecarga imposta ao ventrículo direito pela hipertensão pulmonar prévia e o aumento da resistência vascular pulmonar por adicional vasoconstrição, é compreensível sua rápida e grave descompensação, em face das modificações hemodinâmicas próprias do final da gravidez, particularmente o acréscimo súbito do retorno venoso no periparto e a redução do aporte de sangue ao ventrículo esquerdo. Dessa forma surgem distúrbios circulatórios e hipóxia durante o parto ou no pós-parto, que ocasionam colapso cardiovascular súbito e irreversível. Em tais circunstâncias ocorrem, também, inclusive como causa de morte súbita, arritmias e possivel descarga vagal por aumento do fluxo nas artérias pulmonares proximais, que provoca bradicardia, bloqueio átrio-ventricular, dissociação eletromecânica e redução do débito cardíaco ${ }^{2,3}$.

A hipertensão portopulmonar representa sindrome insuficientemente caracterizada, havendo poucos relatos com grandes séries de casos. A hipertensão portal com ou sem hepatopatia é reconhecida, na maioria dos casos, antes da hipertensão pulmonar, com intervalo médio de cinco a seis anos, evoluindo a última de forma subclínica, por meses ou anos, antes do aparecimento de sintomas, fato que se daria de forma súbita ou progressiva, em geral por disfunção do ventrículo direito ${ }^{11,12}$. No caso 1 a hipertensão portal precedeu, provavelmente, por mais de dez anos as graves manifestações da hipertensão pulmonar no pós-parto, ao passo que no caso 2 esse tempo não ficou definido. O risco de desenvolver hipertensão pulmonar aumenta com a duração e não com a gravidade da hipertensão portal, da insuficiência hepática ou do volume de sangue desviado ${ }^{12,15,16}$.

Descrita pela primeira vez em $1951^{17}$, a hipertensão portopulmonar ocorre em cerca de $1 \mathrm{a}$ $4 \%$ dos pacientes portadores de hepatopatias crônicas, representando $10 \%$ dos casos de hiperten- 
são pulmonar $9,11,12,15,16$. Tornou-se mais reconhecida e valorizada com o advento dos transplantes hepáticos ${ }^{10}$, por constituir contra-indicação relativa ao procedimento. Nos casos bem estabelecidos apresenta perfil hemodinâmico peculiar com características semelhantes à hipertensão pulmonar primária (pressão capilar pulmonar normal, aumento da pressão de artéria pulmonar e da resistência vascular pulmonar); secundariamente contribuiriam a circulação hiperdinâmica, baixa resistência vascular sistêmica, aumento do índice cardiaco e crescimento do fluxo pulmonar, observados na hipertensão portal ${ }^{10-12,15,16,18,19}$.

É considerada, por vezes, erroneamente, dentro do grupo da hipertensão pulmonar primária, talvez pela semelhança do estado funcional cardiopulmonar, das alterações vasculares e a possivel relação patogenética com a vasoconstrição ou por um desequilibrio entre fatores vasoconstritores (serotonina, neuropeptídeo $\mathrm{Y}$, tromboxana) e vasodilatadores (prostaglandinas) pulmonares, em pacientes geneticamente suscetíveis ${ }^{11,12,18}$.

Admite-se que a hipertensão portopulmonar resulte do aporte de substâncias vasoativas, citocinas e fatores de crescimento, provenientes da área esplâncnica, através de shunts portosistêmicos, desviando-se, portanto, do metabolismo hepático. Da remodelação vascular pulmonar participaria a interleucina-1, agregação plaquetária, microtrombos in situ, ocorrendo, ainda, disfunção das células endoteliais, liberação de endotelina e redução do óxido nítrico, que atuam na vasorregulação ${ }^{20}$. A plaquetopenia já descrita anteriormente ${ }^{18}$, cuja causa não está bem definida, foi observada no caso em que foi avaliada (caso 2).

Como fator coadjuvante, em certos casos de hipertensão portopulmonar, pode surgir tromboembolismo pulmonar, especialmente de pequenos vasos, favorecido aqui pelo estado de hipercoagulabilidade reconhecido no puerpério imediato, que agravaria a hipertensão pulmonar. É válido assinalar que gestantes com hipertensão pulmonar, nas quais se associa a pré-eclampsia, o declínio súbito da resistência vascular periférica no pós-parto é mais pronunciado, constituindo fator contribuinte significativo.

As características morfológicas talvez justifiquem a inclusão da hipertensão portopulmonar na arteriopatia pulmonar denominada plexogênica, que abrange, ainda, a que acompanha cardiopatias com shunt (Eisenmenger), a hipertensão pulmonar por drogas (anorexígenos, cocaína), na infecção pelo HIV e a hipertensão pulmonar primária (hipertensão pulmonar plexogênica idiopática). Na verdade, a semelhança seria apenas morfológica, variando a patogênese e cabendo o termo hipertensão pulmonar primá- ria quando a etiologia for absolutamente desconhecida.

As necrópsias revelaram, além do diagnóstico não suspeitado clinicamente de hipertensão pulmonar, hepatopatia fibrosante em ambos os casos, caracterizada por neoformação conjuntiva sistematizada nos espaços portobiliares, envolvendo ramos da veia porta, correspondendo ao relatado na hipertensão portal idiopática. Trata-se de condição com numerosa sinonímia (fibrose portal não cirrótica, cirrose septal incompleta, esclerose hepatoportal, pseudocirrose) e controversa se constitui ou não entidade única ${ }^{21-23}$. É relatada especialmente na Índia e Japão, porém pouco reconhecida em nosso meio.

O quadro anatomopatológico assemelhou-se ao descrito na fibrose hepática esquistossomótica (forma de Symmers), embora estivessem ausentes ovos, granulomas e neoformação vascular, sendo escasso o infiltrado inflamatório. A diferenciação não é fácil, principalmente em zonas endêmicas para esquistossomose mansônica, havendo possivel subestimação da hipertensão portal idiopática, que determina também hipertensão portal pré-sinusoidal ${ }^{21,23}$.

Há possibilidade de a cura parasitológica e controle das reinfecções interferirem no padrão morfológico hepático, falando-se em regressão da forma hepatosplênica ${ }^{24}$, todavia não se conhece, exatamente, quais seriam as modificações histopatológicas e suas repercussões sobre a hemodinâmica portal no homem.

A hipertensão portal não foi reconhecida no curso da gravidez, faltando evidências de circulação colateral porto-sistêmica, relato de episódios de hemorragia digestiva ou sinais de insuficiência hepática. No caso 1 ocorreu esplenectomia prévia, cujo motivo a paciente desconhecia, confirmada pela necrópsia, ao passo que no caso 2 observou-se baço esclerocongestivo e varizes no terço inferior do esôfago.

A hipertensão pulmonar, talvez pelo seu tempo de evolução nos dois casos, não alcançou grau adiantado de lesão vascular, sendo fato estabelecido a ausência de correspondência entre as alterações hemodinâmicas e as anatomopatológicas $^{9}$. Constatou-se hipertrofia da camada média das pequenas artérias, neoformação conjuntiva intimal e lesões plexiformes, acrescidas no caso 2 de obstrução por trombose de pequenos vasos. Verificou-se, portanto, semelhança com o descrito na hipertensão pulmonar primária, sendo que em certos casos, possivelmente iniciais, não se observam as lesões plexiformes ${ }^{5,11}$. A pesquisa exaustiva de ovos e granulomas de $S$. mansoni foi negativa, assim como o padrão de resposta vascular descrito na forma cardiopulmonar da esquistossomose. 
Nos dois casos foram notadas repercussões sobre o ventrículo direito, constituindo cor pulmonale crônico, com dilatação recente das câmaras de enchimento e esvaziamento, representando evidência morfológica de descompensação, resultado das modificações hemodinâmicas bruscas que se verificaram no periparto. Alterações compativeis com o estado de choque, com toda probabilidade cardiogênico, foram identificadas em vários órgãos, especialmente no figado.

O conjunto de evidências indica a presença de hipertensão portopulmonar, representada pela hipertensão portal idiopática, cuja associação com hipertensão pulmonar já foi registrada, inclusive no curso de gravidez ${ }^{8}$. É possivel que a longa sobrevida das pacientes com hipertensão portal idiopática, especialmente se controladas eventuais hemorragias por ruptura de varizes esofagianas, condicionaria o risco de desenvolvimento de hipertensão pulmonar e essa pelo seu curso progressivo acaba sendo responsável pelo óbito, antes da hipertensão portal. O contrário sucede nas pacientes com cirrose hepática, dependendo da sua gravidade, sendo o diagnóstico da hipertensão portopulmonar realizado, por vezes, só com estudos hemodinâmicos ${ }^{15,16,19}$.

Confirmou-se, dessa forma, que a hipertensão pulmonar e gravidez constituem combinação de alto risco, com expressivas taxas de mortalidade materna ${ }^{14}$, embora no caso específico da hipertensão portopulmonar não se tenham informações sobre os índices de mortalidade. Por analogia, como se trata de arteriopatia plexogênica, é possivel que a porcentagem de óbitos se aproxime dos 35 a 50\% assinalados para a hipertensão pulmonar primária e gravidez, não parecendo manter relação com o grau da lesão vascular pulmonar.

Na hipertensão portopulmonar o prognóstico é reservado e depende em grande parte da integridade funcional do ventrículo direito ${ }^{15}$. Os casos relatados comprovam a gravidade do processo quando associado à gravidez, justificando a necessidade de acompanhamento multidisciplinar no pré-natal e periparto em pacientes portadoras de hipertensão pulmonar, cujo reconhecimento deve ser o mais precoce possivel, o que permitiria cuidados durante o parto (decúbito lateral, anestesia epidural segmentar, balanço hídrico adequado), uso eventual de drogas visando aumento do débito cardiaco, vasodilatadores pulmonares e controle da insuficiência cardiaca, objetivando melhor prognóstico ${ }^{1-3,12,25}$.

\section{ABSTRACT}

The severity of the association of pulmonary hypertension with pregnancy is well known. Pulmonary arterial hypertension constitutes one of the highest risk conditions for maternal mortality in late pregnancy and postpartum. Patients with portal hypertension of varying etiology may develop pulmonary arterial hypertension (portopulmonary hypertension) and most cases present cirrhosis as the underlying disease; however, a few cases of noncirrhotic etiology have been described. Clinical and pathological findings in two cases of portopulmonary hypertension and pregnancy are presented here. The two patients (30 and 24 years old) developed severe right heart failure and shock just after the delivery and the disease progressed rapidly to death. Autopsy demonstrated fibrosis in hepatic portal tracts, as has been described in cases of idiopathic portal hypertension. Also, pulmonary hypertension classified as plexogenic was reported.

KEY WORDS: Portal hypertension. Pulmonary hypertension. Portopulmonary hypertension. Pregnancy complications. Maternal mortality.

\section{Referências}

1. Weiss BM, Zemp L, Seifert B, Hess OM. Outcome of pulmonary vascular disease in pregnancy: a systematic overview from 1978 through 1996. J Am Coll Cardiol 1998; 31:1650-7.

2. Weiss BM, Hess OM. Pulmonary vascular disease and pregnancy: current controversies, management strategies, and perspectives. Eur Heart J 2000; 21:104-15.

3. Smedstad KG, Cramb R, Morison DH. Pulmonary hypertension and pregnancy: a series of eight cases. Can J Anaesth 1994; 41:502-12.

4. Avila WS, Grinberg M, Snitcowsky R, et al. Maternal and fetal outcome in pregnant women with Eisenmenger's syndrome. Eur Heart J 1995; $16: 460-4$.

5. Dawkins KD, Burke CM, Billingham ME, Jamieson SW. Primary pulmonary hypertension and pregnancy. Chest 1986; 89:383-8.

6. Ray J, Sermer M. Systemic lupus erythematosus and pulmonary hypertension during pregnancy: report of a case fatality. Can J Cardiol 1996; 12:753-6.

7. Powars DR, Sandhu M, Niland-Weiss J, Johnson C, Bruce S, Manning PR. Pregnancy in sickle cell disease. Obstet Gynecol 1986; 67:217-28. 
8. Goenka MK, Mehta SK, Malik AK, Kumar YR, Kochhar R. Fatal pulmonary arterial hypertension complicating noncirrhotic portal fibrosis. Am J Gastroenterol 1992; 87:1203-5.

9. Krowka MJ. Hepatopulmonary syndrome versus portopulmonary hypertension: distinctions and dilemmas. Hepatology 1997; 25:1282-4.

10.Kuo PC, Plotkin JS, Johnson LB, et al. Distinctive clinical features of portopulmonary hypertension. Chest 1997; 112: 980-6.

11. Hervé P, Lebrec D, Brenot F, et al. Pulmonary vascular disorders in portal hypertension. Eur Respir J 1998; 11:1153-66.

12.Mandell MS, Groves BM. Pulmonary hypertension in chronic liver disease. Clin Chest Med 1996; 17:17-33.

13.Schraufanagel DE, Kay JM. Structural and pathologic changes in the lung vasculature in chronic liver disease. Clin Chest Med 1996; 17:1-15.

14.Roberts NV, Keast PJ. Pulmonary hypertension and pregnancy: a lethal combination. Anaesth Intensive Care 1990; 18:366-74.

15.Hadengue A, Benhayoun MK, Lebrec D, Benhamou JP. Pulmonary hypertension complicating portal hypertension: prevalence and relation to splanchnic hemodynamics. Gastroenterology 1991; 100:520-8.

16. Castro M, Krowka MJ, Schroeder DR, et al. Frequency and clinical implications of increased pulmonary artery pressures in liver transplant patients. Mayo Clin Proc 1996; 71:543-51.
17. Mantz FA, Graige E. Portal axis thrombosis with spontaneous portacaval shunt and resultant cor pulmonale. AMA Arch Pathol 1951; 52:91-7.

18.Edwards BS, Weir EK, Edwards WD, Ludwig J, Dykoski RK, Edwards JE. Coexistent pulmonary and portal hypertension: morphologic and clinical features. J Am Coll Cardiol 1987; 10:1233-8.

19.Yang YY, Lin HC, Lee WC, et al. Portopulmonary hypertension: distinctive hemodynamic and clinical manifestations. J Gastroenterol 2001; 36:181-6.

20.Panos RJ, Baker SK. Mediators, cytokines, and growth factors in liver-lung interactions. Clin Chest Med 1996; 17:151-69.

21.Sarin SK. Non-cirrhotic portal fibrosis. Gut 1989; 30:406-15.

22.Ludwig J, Hashimoto E, Obata H, Baldus WP. Idiopathic portal hypertension: a histopathological study of 26 Japanese cases. Histopathology 1993; 22:227-34.

23.Nakanuma Y, Tsuneyama K, Ohbu M, Katayanagi K. Pathology and pathogenesis of idiopathic portal hypertension with an emphasis on the liver. Pathol Res Pract 2001; 197:65-76.

24.Bina JC, Prata A. Regressão da hepatosplenomegalia pelo tratamento específico da esquistossomose. Rev Soc Bras Med Trop 1983; 16:213-8.

25. Stewart R, Tuazon D, Olson G, Duarte AG. Pregnancy and primary pulmonary hypertension: successful outcome with epoprostenol therapy. Chest 2001; 119:973-5.

\section{RBGO}

\section{É uma publicação da FEBRASGO}

que aceita artigos provenientes de ginecologistas,

obstetras e de outras especialidades. Portanto, publique!!!

Mande já seu artigo para RBGO 